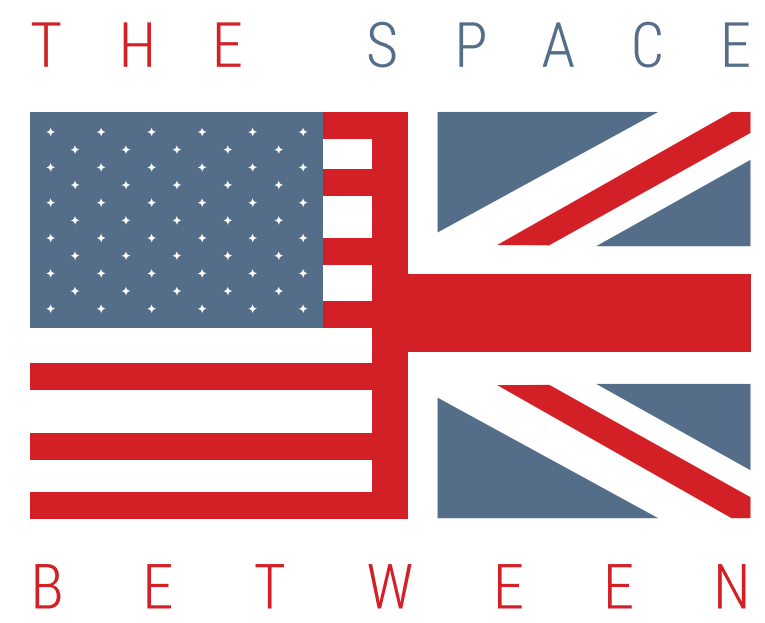

$$
\begin{gathered}
R O G \quad E R \quad C . S C H O N F E L D \\
\text { K A T E W U L F S O N }
\end{gathered}
$$

ITHAKA S+R 


\section{THE SPACE BETWEEN}

Ithaka $\mathrm{S}+\mathrm{R}$ has been tracking the attitudes and practices of faculty members at United States colleges and universities for over a decade. Our triennial survey focuses on evolving research methods and pedagogies and the impacts these changes are having on service needs such as those provided by academic libraries and learned societies. Last year, in partnership with Jisc and RLUK, we ran a parallel survey in the United Kingdom, the first adaptation of our US survey for a different national context. Reports of findings and data sets for both the US Faculty Survey and the UK Survey of Academics are publicly available. ${ }^{1}$ We now have an opportunity to compare the two to understand where there is alignment and where they diverge at a national level, fueling questions as to why differences exist and what implications may arise.

Of course, there are a variety of well known national differences between the US and UK higher education systems. The US population is five times greater than the UK, and has a larger number of institutions operating within a comparatively decentralized structure. It is no surprise therefore that the demographic makeup of our two samples varied in several important ways. In the US, we received 5,261 responses, yielding an aggregate response rate of 3.5\%. In the UK, we received 3,498 responses, for a response rate of $7.9 \%$. In the US project, we included faculty members from three disciplinary groupings in our sample: Humanities, Social Sciences, and Sciences. For the UK project, we added Medical and Veterinary academics as well. To enable comparison, these UK Medical and Veterinary academics are excluded from the analysis in this paper. Even with the elimination of this group, the UK respondents contain a higher proportion of scientists than that of the US. Given the importance of disciplines in response patterns to many questions, the different disciplinary populations are an important driver of many UK-US aggregate differences. But differences that are ultimately disciplinary rather than national are not our focus here.

So are there national differences? When taken as a whole, the data from each survey are remarkably consistent. This suggests that despite the structural and institutional differences between the higher education sectors in the UK and the US, when it comes to the digital environment, the practices and behaviors of researchers and teachers are, in many cases, quite similar. However, we do see some areas where there is clear divergence.

\section{ACCESS}

One of the most important differences between the UK and US data relates to a series of questions that asked how academics gain access to different types of material. When asked how they access journal articles and monographs they routinely use, a modestly higher share of UK respondents reported relying on their own library's collections or subscriptions. By contrast, a larger share of US respondents reported that they often supplement their own library's collections with materials borrowed from other institutions through services such as inter-library loan.

\section{When asked specifically about journal articles not in}

their own library's collections, a remarkably larger share

of US respondents strongly agreed that they can "always

get satisfactory access to these materials elsewhere." 
Figure 1: "When you think about the journal articles and scholarly monographs that you routinely use - for research as well as for teaching - how important are each of the following sources?” Percent of respondents who indicated that each of these sources is very important.

My college or university library's collections or subscriptions

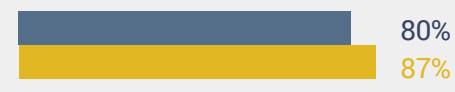

Materials that are freely available online

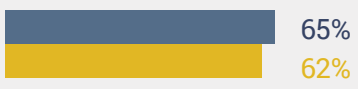

My own personal collections or subscriptions

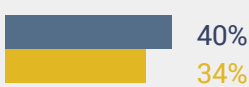

Collections or subscriptions of other institutions

My academic department's collections or subscriptions

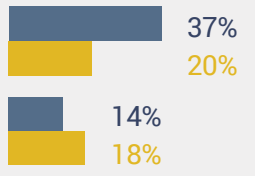

$65 \%$

Given their higher degree of reliance on their own institutions, it is not surprising that UK academics have more trouble gaining access to materials that are "not immediately available" through their library's collections and subscriptions. As with routine items, a large share of US scholars reported using inter-library loan or document delivery services, while a much smaller share of UKacademics reported extending their access in this way. By contrast, a majority of UK respondents reported that when they do not have immediate access to needed material, they regularly "give up and look for a different resource." Though not a majority, a high share of UK respondents reported using relatively informal methods such as contacting the author, or asking a friend at another institution. More than 4 out of 5 respondents from both surveys reported that they regularly "search for a freely available version online," although a larger share of UK respondents reported that they "often" use this method. When asked specifically about journal articles not in their own library's collections, a remarkably larger share of US respondents strongly agreed that they can "always get satisfactory access to these materials elsewhere."

Figure 2: "When you want a scholarly monograph or journal article that you do not have immediate access to through your college or university library's collections, how often do you use each of the following methods to seek access to that material often, occasionally, rarely, never?" Percent of respondents who indicated that they "often" or "occasionally" use each method to access material.

Search for a freely available version online

Use interlibrary loan or document delivery service provided by my library

Give up and look for a different resource that I can access

Ask a friend at another institution

Contact the author

Purchase it myself from the publisher or a vendor
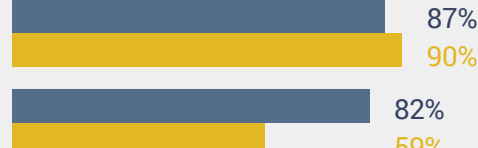
$59 \%$

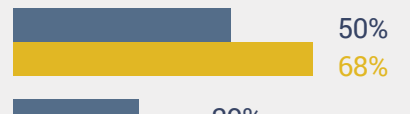

$68 \%$

$40 \%$

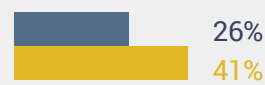

$41 \%$

$33 \%$

$36 \%$ 


\section{E-BOOKS}

A second area where the data reveal a notable difference is in the use of e-books. To understand both practices and preferences associated with electronic monographs, we asked academics how frequently they use e-books in their research, as well as whether they consider e-books to "play a very important role" in their research. In both cases, a smaller share of UK respondents reported either regularly using e-books, or viewing them as important to their work. Interestingly, on the question of perceived importance, the US data reveal a disciplinary pattern that is not mirrored in the UK. Among US respondents, a lower share of humanists reported that e-books are very important to their research, compared to higher shares of both scientists and social scientists. The UK data reveal more consistency, as the share of respondents in each disciplinary grouping who view e-books as very important is virtually the same.

Figure 3: Percent of respondents who strongly agree with the statement: "Electronic versions of scholarly monographs play a very important role in my research," by disciplinary grouping.

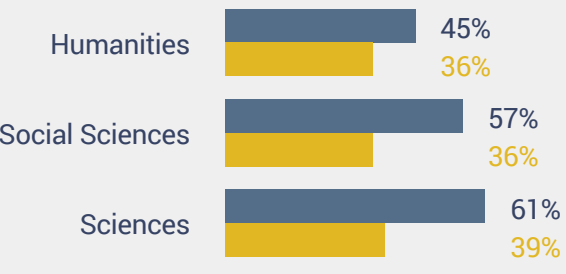

Figure 4: "You may have the opportunity to read scholarly monographs in electronic format, either through a library subscription database or as standalone e-books. How often have you used scholarly monographs in digital form in the past six months - often, occasionally, rarely, or never?" Percent of respondents who indicated that they have "often" or "occasionally" used e-books in the last six months.

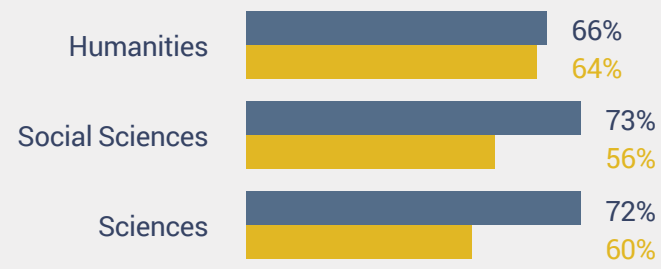

In considering a variety of use cases for scholarly monographs from exploration to long-form reading, UK academics revealed a greater preference for print versions; for each activity, the share of UK academics who indicated it is easier in print was higher, and the share indicating it is easier digitally is smaller. When asked about features that might improve the value of e-books, such as improved navigation and annotation tools and increasing the number available, a smaller proportion of UK academics agreed that each feature would make e-books more valuable. ${ }^{3}$ 


\section{TECHNOLOGY-ENABLED RESEARCH AND INSTRUCTION}

Our survey contained several questions designed to understand how academics are incorporating technology enhanced methods into their teaching and research. Here the data reveal a disparity that is relatively small yet strikingly consistent. For virtually every question, a slightly smaller share of UK academics reported engaging with technology enhanced research methods, and this held constant across a variety of question types and phrasing. We asked academics about a number of research methods including GIS/ mapping, analysis of quantitative data, writing code, and the use of models or simulations. For some methods, such as text mining and the analysis of pre-existing data, the difference between the US and UK data is substantial, while for other methods the gap is not meaningful. The only research method rated very important by a higher share of UK academics was "writing software or code," and here the difference was only $1 \%$.

For virtually every question, a slightly smaller

share of UK academics reported engaging

with technology enhanced research methods, and this held constant across a variety of question types and phrasing.

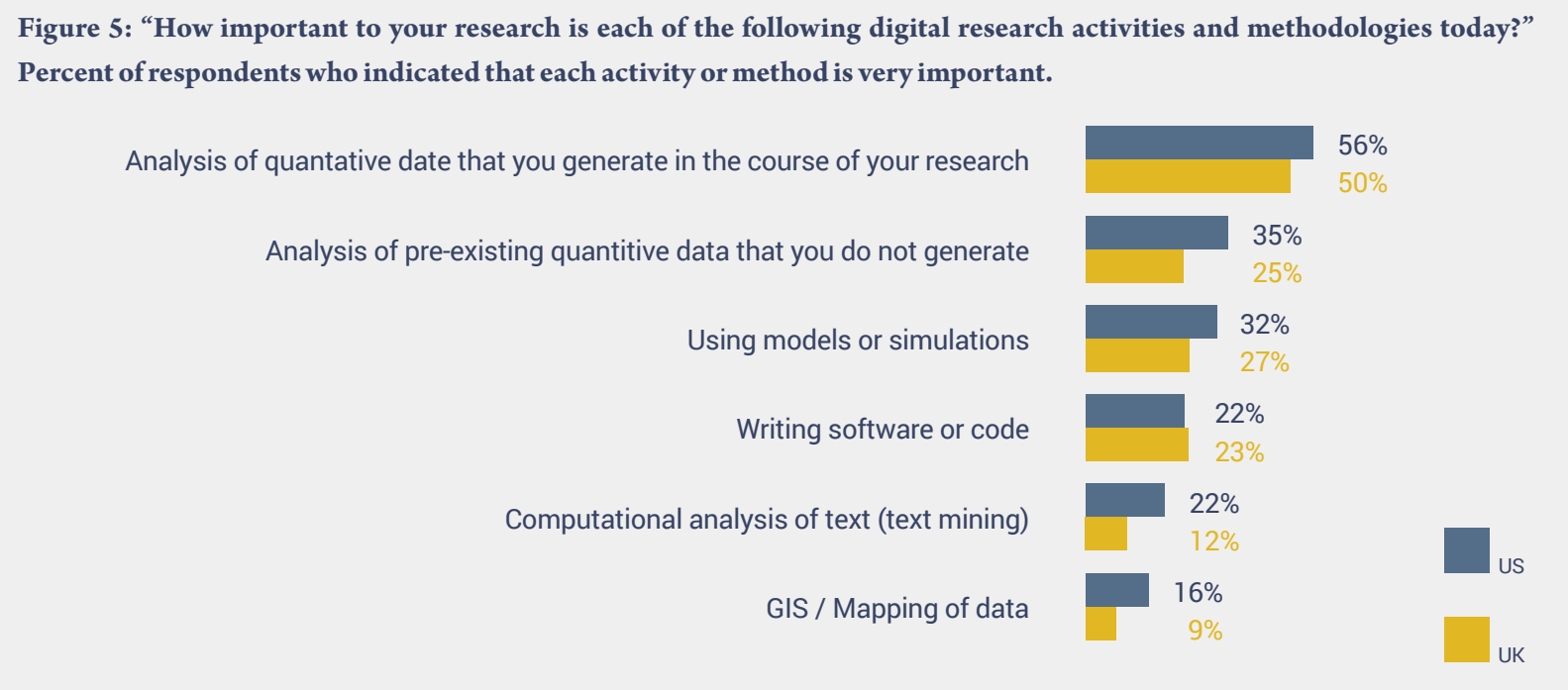

Across the board, the data reveal that social scientists account for much of this difference. Notably higher shares of social scientists in the US than in the UK indicated that analyses of quantitative data are very important research methods. 


\section{Notably higher shares of social scientists in the US}

\section{than in the UK indicated that analyses of quantitative}

\section{data are very important research methods.}

Figure 5: "How important to your research is each of the following digital research activities and methodologies today?" Percent of respondents who indicated that each activity or method is very important (social scientists only).

Analysis of quantative date that you generate in the course of your research

Analysis of pre-existing quantitive data that you do not generate

Using models or simulations

Writing software or code

Computational analysis of text (text mining)

GIS / Mapping of data

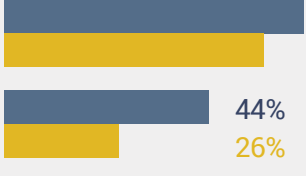

$64 \%$

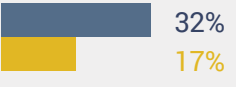

$17 \%$

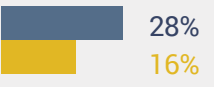

$16 \%$

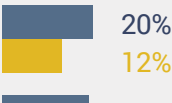

$12 \%$

$19 \%$

$10 \%$

Compared to research, smaller shares of both US and UK academics reported using digitally enhanced teaching methods. ${ }^{13}$ The only teaching method used regularly by more than half of both UK and US respondents was the relatively established practice of showing videos in the classroom to supplement other forms of learning. For some teaching enhanced methods, the US and UK numbers are virtually identical, while for others, the share of US academics using the method was notably larger (supplement in-class time with additional audio or video modules, assigning students to create audiovisual or digital media projects, and assigning students to share reading responses on a course discussion board). In two cases, the UK numbers are higher, but only by very small margins (make audio or video lectures available online, and the use of automated online tools to evaluate student problem sets). Not only do US faculty members report using more digitally enhanced methods, they also expressed a greater interest in further integrating digital methods into their work; here again this gap is highest among social scientists. 


\section{RESEARCH DISSEMINATION}

We asked respondents about their publishing frequency in a variety of formats, and with the sole exception of trade books, a larger share of UKacademics reported "often" or "occasionally" publishing their work in each kind of format. In this case, the difference may be due to a combination of the greater average research intensity of the UK higher education sector as well as the comparatively higher proportion of scientists in the UK sample.

\section{....with the sole exception of trade books, a}

\section{larger share of UK academics reported "often"}

\section{or "occasionally" publishing their work in each}

\section{kind of format.}

Figure 7: "You may have had the opportunity to share the findings of your scholarly research in a variety of different formats. Please...indicate how often you have shared the findings of your scholarly research in each of the following ways in the past five years - often, occasionally, rarely, or never." Percent of respondents who indicated that they have "often" or "occasionally" shared their findings in each format.

\section{Peer-reviewed journals \\ Published conference proceedings}

Scholarly monographs or edited volumes, published by an academic

Digital publications other than the types of publication options listed

Magazines and trade journals that are not peer reviewed

Blogs or social media

Trade books that do not specifically target an academic audience
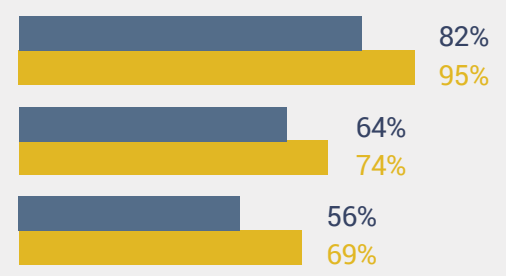

$31 \%$

$30 \%$

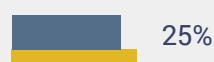

$30 \%$

$19 \%$

$21 \%$

$18 \%$

$16 \%$ 


\section{CONCLUDING QUESTIONS}

This comparative analysis raises a number of significant questions with real policy implications.

What accounts for the apparent methodological divergence between UK and US social scientists with respect to quantitative approaches? Is this explained by different incentives, skills, interests, or other factors? This set of issues and underlying trends may be particularly relevant to universities, funding agencies, and policy-makers.

What accounts for comparatively higher levels of adoption of e-books in the US and comparatively lower levels in the UK? Is this driven by different levels of availability, different features, services, or support, different essential preferences, or other factors? Content providers operating internationally may wish to consider these issues, and what trends may emerge.

The UK higher education sector is comparatively more centralized than is the US, in many ways. It was therefore counter-intuitive to find comparatively higher level of reliance on institutional library collections in the UK, where more sharing might be expected, and comparatively higher levels of reliance on other sources in the US, where more self-reliance might be expected. Is this an indication of improvements needed to interlibrary lending or document supply, or further work to improve or facilitate access to research materials, in the UK? Is this an indication that smaller institutions in the US do not seek to provide self-sufficient collections? Libraries and content providers alike may wish to consider these issues in planning strategy and services.

These questions offer a first look at some of the comparative insights that emerge from a close reading of the US and UK surveys. Our analysis of these data will continue, and we encourage further analysis from others across the community, the sharing of questions it raises for you, and thoughts about the reasons for divergence and the implications. 


\section{END NOTES}

I The reports are freely available for download at http://www.sr.ithaka.org/research-publications/us-faculty-survey-2012 and http://www.sr.ithaka.org/research-publications/ ithaka-sr-jisc-rluk-uk-survey-academics-2012. Datasets for Ithaka S+R's surveys are being made available through ICPSR, where they may be found at icpsr.umich.edu/ icpsrweb/ICPSR/series/226

${ }^{2} 59 \%$ of US respondents strongly agreed with the statement "When I discover journal articles I need for my research that are not in my library's collections, I can almost always get satisfactory access to these materials elsewhere," compared with $37 \%$ of UK respondents.

${ }^{4}$ For "reading cover to cover in depth", $88 \%$ of UK respondents reported that this is easier in print, and $4 \%$ reported that it is easier in digital, compared to $81 \%$ of US respondents who reported that it is easier in print, and 6\% who reported that it is easier in digital; for "reading a section in depth", $69 \%$ of UK respondents reported that this is easier in print, and $8 \%$ reported that it is easier in digital, compared to $61 \%$ of US respondents who reported that it is easier in print, and $12 \%$ who reported that it is easier in digital; for "skimming in whole or in part," $46 \%$ of UK respondents reported that this is easier in print, and $30 \%$ reported that it is easier in digital, compared to $40 \%$ of US respondents who reported that it is easier in print, and $32 \%$ who reported that it is easier in digital; for "searching for a particular topic", $16 \%$ of UK respondents reported that this is easier in print, and $71 \%$ reported that it is easier in digital, compared to $15 \%$ of US respondents who reported that it is easier in print, and $73 \%$ who reported that it is easier in digital; for "exploring references," $18 \%$ of UK respondents reported that this is easier in print, and $59 \%$ reported that it is easier in digital, compared to $15 \%$ of US respondents who reported that it is easier in print, and $61 \%$ who reported that it is easier in digital; for "comparing treatment of ideas between monographs", $47 \%$ of UK respondents reported that this is easier in print, and $17 \%$ reported that it is easier in digital, compared to $43 \%$ of US respondents who reported that it is easier in print, and $21 \%$ who reported that it is easier in digital. Regarding factors that would make e-books more valuable, $71 \%$ of UK respondents reported that "access to a wider range of materials in digital form" would make e-books more valuable, compared to $79 \%$ of US respondents; $63 \%$ of UK respondents reported that "improved ability to download and organize a personal collection of monographs" would make e-books more valuable, compared to $73 \%$ of US respondents; $65 \%$ of UK respondents reported that "improved ability to navigate through and among monographs" would make e-books more valuable, compared to $75 \%$ of US respondents; $57 \%$ of UK respondents reported that "Improved ability to read academic monographs on my device of choice" would make e-books more valuable, compared to $64 \%$ of US respondents; $66 \%$ of UK respondents reported that "improved ability to highlight, annotate, and print materials as needed" would make e-books more valuable, compared to $72 \%$ of US respondents; $46 \%$ of UK respondents reported that "the ability to perform computational analysis (text mining) over a corpus of electronic monographs" would make e-books more valuable, compared to $56 \%$ of US respondents; $48 \%$ of UK respondents reported that "more effective integration of images, multimedia, and graphs linked to the text" would make e-books more valuable, compared to $65 \%$ of US respondents; $43 \%$ of UK respondents reported that "certified preservation of digital academic monographs" would make e-books more valuable, compared to $52 \%$ of US respondents.

${ }^{13}$ We asked about the following digitally enhanced teaching methods: "Make audio or video of my lectures available online for my students to access (27\% US, $28 \%$ UK); "make audio or video of my lectures available online for the general public," (8\% US, 8\% UK); "supplement in-class time with additional audio or video modules," (38\% US, $20 \%$ UK); "rely on students watching my lectures through recorded audio or video to reserve face to face time for other activities," ( $9 \%$ US, $5 \%$ UK); "ask my students to meet with each other through voice or video chat," (18\% US, 13\% UK); "voice or video chat with students one-on-one or in small groups," (12\% US, $6 \%$ UK); "use automated online tools to evaluate student problem sets and offer feedback or guidance in real time to students," (31\% US, 35\% UK); "use publisher-provided instructional modules that accompany a textbook," (35\% US, $15 \%$ UK); "assign students to create audiovisual or digital media projects," (41\% US, 24\% UK); "assign students to share reading responses on a course discussion board," (39\% US, $22 \%$ UK); "use digital games or simulations to allow students to explore concepts," (21\% US, $13 \%$ UK); "show videos in the classroom, instead of or as one component of a lecture or discussion," (67\% US, $54 \%$ UK); "use social media (such as Facebook or Twitter) to keep in touch with students currently enrolled in your courses," (13\% US, 10\% UK). 